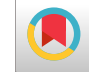

\title{
Posttraumatic Stress Symptoms and General Mental Health Problems During the COVID-19 Pandemic in Iran: A Web-Based Cross-sectional
} Survey

\author{
Razieh Salehian (iD ${ }^{1}$, Atefeh Ghanbari Jolfaei ${ }^{1}$, Morteza Naserbakht ${ }^{2}$ and Mahnaz Abdi ${ }^{1,{ }^{*}}$ \\ ${ }^{1}$ Rasoul-e-Akram Hospital, Iran University of Medical Sciences, Tehran, Iran \\ ${ }^{2}$ Mental Health Research Center, Psychosocial Health Research Institute, Iran University of Medical Sciences, Tehran, Iran \\ "Corresponding author: Rasoul-e-Akram Hospital, Iran University of Medical Sciences, Tehran, Iran. Email: heroabdi@yahoo.com
}

Received 2021 March 08; Revised 2021 July 03; Accepted 2021 September 02.

\begin{abstract}
Background: The COVID-19 pandemic as a stressor can harm the community's mental health. Iran is one of the first countries to be severely affected by COVID-19 since February 2020.

Objectives: This study aimed to assess the rates of COVID-19-related Posttraumatic Stress Symptoms (PTSS) and the general mental health burden among the Iranian population during the pandemic and to explore the potential influencing factors.

Methods: Through a web-based cross-sectional survey, based on social media, data were collected from self-selected volunteers using a demographic information form, General Health Questionnaire-28 (GHQ-28), and Impact of Event Scale-Revised (IES-R).

Results: Among 1,910 analyzed respondents, the overall prevalence of COVID-19-related PTSS and general mental health burden was 62.4 and $43.6 \%$, respectively. Regarding mental health, the burden was greater in the social and anxiety dimensions than in the physical and depression dimensions. The prevalence of PTSS was higher in women, younger age groups, divorced/widowed individuals, people with a history of psychiatric disorders, and those who had experienced other stressful events in the last year (P-values $<0.05$ ). Multivariable logistic regression showed that a positive history of other stressful events and the GHQ-28 score were the potential influencing factors associated with PTSS (AOR = 2.468 and 6.007, respectively; P-values $<0.001$ ).

Conclusions: The study identified a significant mental health burden and PTSS among Iranians during the COVID-19 pandemic. Continuous assessment and monitoring of the psychological consequences of pandemics should be considered in Iran and other countries.
\end{abstract}

Keywords: Coronavirus Disease 2019, Mental Health, Trauma, Stress

\section{Background}

In the 21st century, multiple outbreaks such as Severe Acute Respiratory Syndrome (SARS) and Middle East Respiratory Syndrome (MERS) have occurred in the world, and recently the people of the world have encountered one of the most contagious outbreaks called Coronavirus Disease 2019 (COVID-19), which quickly became a pandemic (1). In Iran, the first COVID-19 case was reported in Qom on 19 February 2020 (2), and at this time, the World Health Organization (WHO) declared COVID-19 a public health emergency threatening all the people of the world (1).

As known, COVID-19 is a highly contagious disease caused by a novel coronavirus that can lead to hospitalization and even death. Compared to previous infectious epidemics, COVID-19 has been more widespread, and in many countries, unprecedented efforts have been made to prac- tice social distancing and to change national behavioral patterns (3). In addition, the global outbreak of COVID-19 can have serious psychological impacts on the communities, and studies of epidemics show that the psychological effects go beyond the fear of contracting the virus (4).

In the current pandemic, there are reasons to speculate that the psychological condition of society may be affected during the infectious disease outbreak (5). Studies conducted during the COVID-19 pandemic in other countries, including China (6), Saudi Arabia (7), and Italy (8), have shown that the current pandemic has had significant psychological effects (anxiety symptoms, depressive symptoms, and post-traumatic stress symptoms) on the general population, and these mental health problems were significant predictors of COVID-19-related PTSD (9). A systematic review that evaluated the rate of stress, anxiety, and depression among the general population during the COVID- 
19 pandemic in different continents (10) reported that the prevalence of anxiety and depression was higher in Asia than in Europe (32.9 and 35.3\% versus 23.8 and 32.4\%, respectively) but the prevalence of stress was lower in Asia than in Europe (27.9 versus 31.9\%). Therefore, the COVID-19 pandemic represents a threatening and potentially traumatic event that can lead to a mental health burden, and it seems that the nature and the effects of COVID-19 on individuals may vary from a country to another as various factors can affect it. Because the mental health burden and PTSS are the predictors of PTSD, the continuous monitoring of the psychological consequences of the COVID-19 outbreak is essential to avoid long-term consequences. In Iran, few studies have been conducted to examine the psychological effects of the COVID-19 pandemic in the general population.

\section{Objectives}

This study was conducted to assess the COVID-19related PTSS and its general mental health burden and to explore the potential influencing factors in the Iranian population. It is hoped that the findings could provide data for targeted interventions on the psychological health of the Iranian population during and after the COVID-19 pandemic.

\section{Methods}

\subsection{Study Design and Participants}

This descriptive-analytical cross-sectional study was carried out among Iranian people over the age of 18 living in all 31 provinces of Iran in 2020. To collect data, a web-based survey using social tools was used in an attempt to prevent the spread of the COVID-19 through droplets or contact and considering the fact that, according to international reports, more than 49 million Iranian users in 2017 had access to the internet (11). The questionnaires were distributed to various social media, including WhatsApp and Telegram, through several groups and channels in various provinces. It is noteworthy that this sampling method has been used in many studies during the current pandemic $(6,11,12)$. The participants were informed of the purpose of the study. To encourage the potential respondents to participate in the survey, it was announced that they would receive a report of their mental health condition after completing the questionnaire.

\subsection{Data Collection}

This questionnaire was online for three months, from 20 March 2020. All subjects answered the questionnaires anonymously. Participants were asked to answer the questionnaire items with regard to their experience of the COVID-19 pandemic and indicate how much they had been bothered by each problem. To ensure the quality of the survey, some limitations were set: (1) the participant must be over 18 years of age; (2) each questionnaire could be completed only once per device; (3) some items needed to be answered in reverse; (4) answering some items was mandatory to be able to get the results of their mental health status; and (5) the questionnaires completed in less than two minutes or more than 60 minutes were excluded from the analysis.

\subsection{Ethical Statement}

This study was approved by the Ethics Committee of the Iran University of Medical Sciences, Tehran, Iran (code: 1399.011). All respondents were first asked to read an informed consent form and would enter the survey only if they agreed.

\subsection{Measures}

\subsubsection{Demographic Information}

The first part of the questionnaire collected sociodemographic data. The demographic variables included age, gender, marital status, education level, occupation, income, history of psychiatric disorder, history of COVID-19 in participants or their family members, history of staying at home as recommended during the pandemic, and history of other stressful events during the last year prior to the pandemic.

\subsubsection{General Health Questionnaire-28 (GHQ-28)}

The GHQ-28 is a self-administered instrument widely used as the screening tool for mental disorders in the community (13). It is divided into four subscales, including somatic symptoms, anxiety and sleep disorder, social dysfunction, and depression (14). Each item was scored using a Likert scoring system (from 0 to 3), with a total maximum score of 84 and a minimum of zero. Psychiatric morbidity was defined as a total score of $\geq 23$, and the score of $\geq 7$ in each subscale was defined as psychiatric morbidity in that subscale (15). Studies on the validation of the GHQ-28 have demonstrated its high validity and reliability in different countries (13). The Persian version of GHQ-28 has shown good internal consistency (Cronbach's alpha $=0.70-0.90)$ (16).

\subsubsection{Impact of Event Scale-Revised (IES-R)}

The COVID-19-related posttraumatic morbidity was determined by the IES-R, which is a self-report measure designed to assess subjective distress for a specific traumatic 
stressor in the past week. It has 22 items that measure the symptoms of intrusion, avoidance, and hyper-arousal. Each item is rated on a five-point Likert scale ranging from 0 (not at all) to 4 (extremely). The total score has a range of 0 to 88 . The cutoff point of 33 out of 88 on the total IES-R score is considered to be indicative of a high likelihood of the presence of PTSD (17). In this study, to detect the presence of PTSS rather than diagnostic PTSD, we used a total score of 24 or greater as a cutoff of clinical stress in the community based on other studies $(17,18)$. The Persian version of IES-R has shown good reliability and validity (19).

\subsubsection{Statistical Analysis}

First, descriptive analyses were conducted to describe the socio-demographic characteristics of the participants. Second, the prevalence of mental health burden and COVID-19-related PTSS was analyzed both in general and in terms of variables, and the Pearson chi-square test $\left(\chi^{2}\right)$ was used to compare the differences between the groups. Third, bivariate and multivariable logistic regression models were performed to explore the potential influencing factors on PTSS and general mental health burden. All variables, as well as IES-R and GHQ-28 scores, were entered in the multivariable logistic regression model. The odds ratio (OR), Adjusted odds ratio (AOR), and 95\% confidence interval $(95 \% \mathrm{CI})$ were obtained. All data were analyzed using the Statistical Package for Social Sciences (SPSS) version 24.0. The P values of less than 0.05 were considered statistically significant (two-sided tests).

\section{Results}

\subsection{Demographic Characteristics}

About 6,500 people visited the questionnaire, but1,950 completed it (response rate $=30 \%$ ). Finally, after applying the exclusion criteria, 40 questionnaires were removed, and 1,910 (97.9\%) were included in the study, which were from 31 provinces. The response rate was also calculated according to the population of each province. Except for the capital city Tehran, which had a higher response rate, the response rate in other provinces was commensurate with the population of that province (about $18.9 \%$ of the total participants were from Tehran, while this province accounted for $16.6 \%$ of the country's population, but the response rate in other provinces was proportional to the population of that province). It is noteworthy that Tehran is a place in which different ethnicities and races with different cultures live. Out of 1,950 answered questionnaires, 1,910 with a respondents' mean age of 35.54 years (SD = 9.03) were included in the analysis. Of these respondents, 942 (49.3\%) were men, and 1,185 (62\%) were married. The socio-demographic characteristics of the participants are shown in Table 1.

\subsection{Prevalence of General Mental Health Burden During COVID- 19 Pandemic}

The overall prevalence of the general health burden (total GHQ-28 score $\geq 23$ ) was $43.6 \%$, and the mean total GHQ-28 score in the Iranian population was 23.02 (SD = 13.30). In terms of the subscales of the GHQ-28, the burden was greater in the social and anxiety dimensions than in the physical and depression dimensions (65.8 and $39.8 \%$ versus 36.8 and $21.9 \%$, respectively) (Table 2 ). The findings of the prevalence of general mental health burden stratified by variables are shown in Table 3.

\subsection{Prevalence of PTSS During COVID-19 Pandemic}

The total prevalence of PTSS with a cutoff of 24 was 62.4\%. The mean COVID-19-related PTSS score according to the total IES-R score was $29.32(S D=17.41)$, and the mean scores of its subscales including hyper-arousal, avoidance, and intrusion were 7.40 (SD = 5.77), 11.65 (SD = 6.68), and $10.26(\mathrm{SD}=7.15)$, respectively (Table 2 ). The findings of the prevalence of PTSS stratified by variables are shown in Table 3.

\subsection{Association of Influencing Factors with PTSS and General Mental Health Burden During COVID-19 Pandemic}

The associations of potential influencing factors with COVID-19-related PTSS and general mental health burden are presented in Table 4 . In the multivariable logistic regression model, women were more likely to have a general mental health burden than men $(\mathrm{AOR}=1.387,95 \% \mathrm{CI}: 1.086$ - 1.770). Besides, participants who had a positive history of psychiatric disorder $(\mathrm{AOR}=2.266,95 \% \mathrm{CI}: 1.680-3.058)$, other stressful events, and IES-R $\geq 24$ (AOR $=6.013,95 \%$ CI: 4.725 - 7.653) were more likely to develop general mental health burden during the COVID-19 pandemic. In this model, a positive history of other stressful events and GHQ$28 \geq 23$ were the only potential factors associated with COVID-19-related PTSS.

\section{Discussion}

This study aimed to determine the general mental health burden and the level of COVID-19-related traumatic distress among the Iranian population. The study showed that in $43.6 \%$ of people, general mental health is somehow involved, and about $62.4 \%$ of the participants had scores equal to or greater than 24 on the IES-R, which is the diagnostic requirement for PTSS. 


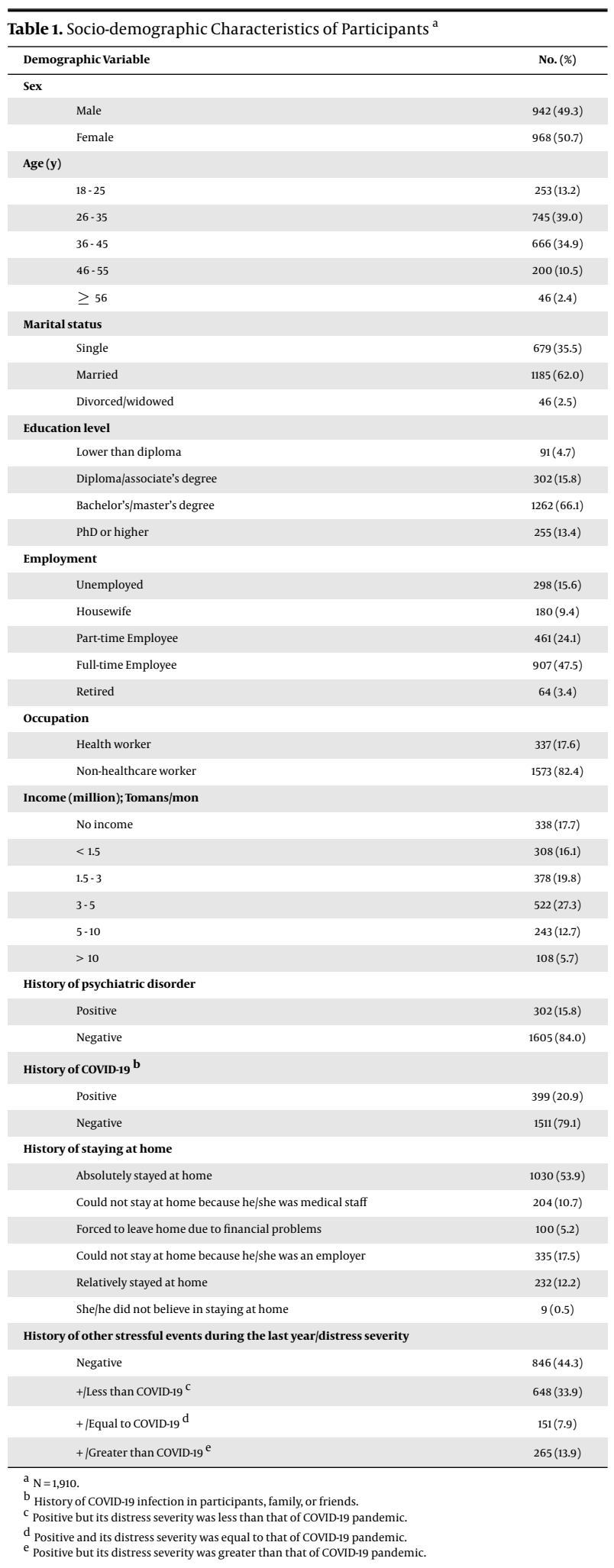

Concerning other studies (6-8), the prevalence of PTSS, anxiety, and depression was higher in Iranians than in other countries, but due to differences between studies, this is not certain. China was the first country to engage with COVID-19. Based on a web-based cross-sectional survey conducted on 603 Chinese participants with an age range of 6 to 80 years (6), the prevalence of anxiety and depression was higher in the Iranian population than in the Chinese one $339.8 \%$ and $21.9 \%$ compared to $34.0 \%$ and $18.1 \%$, respectively); but this is not conclusive because of the differences between the two studies such as the measurement tools (GAD-7 and CES-D), time and duration of the study, and the number of participants.

Another study carried out about the impact of the COVID-19 pandemic on mental health among 18,147 people from the general population in Italy showed that the endorsement rates for PTSS, depression and anxiety were 37, 17.3 , and $20.8 \%$ by using GPS-PTSS, PHQ-9, and GAD-7, respectively (8). Again, the prevalence of PTSS, anxiety and depression was higher in Iranians $(62.4,39.8$, and $21.9 \%$, respectively), although the measuring tools were different. In a study in Saudi Arabia that investigated the psychological impact of the COVID-19 pandemic on 1,160 people from the general population, the average score of the participants on the IES-R questionnaire was 20.9 ( $S D=15.7)$; meanwhile, 28.3 and $24.0 \%$ of the participants had depressive and anxiety symptoms, respectively (by using DASS-21) (7). According to our results, Iranians had more PTSS (mean score of IES-R was 29.32 with $S D=17.41$, and the total prevalence of PTSS was 62.4\%) than the Saudi Arabia population. Also, compared to the mentioned study, our findings revealed higher anxiety but lower depressive symptoms (21.9 and $39.8 \%$ for depressive and anxiety symptoms, respectively).

Comparing the results of the current study with an epidemiological survey conducted on 35,813 people of the Iran general population in 2017 (with an age range of 10 to over 70 years) by using the GHQ-28 (20), an increase was observed in the mental health burden (43.6 versus $23.4 \%$ ); regardless of the study method, this growth rate may be in favor of the negative effects of the COVID-19 pandemic on the mental health of people. Iran is one of the countries whose people have experienced many stressful events in recent years, such as floods, earthquakes, and economic sanctions. The higher rate of mental health problems in Iran compared to other countries and also its doubling in recent years may be due to the cumulative negative effects of the stressful events on mental health $(21,22)$. As the current study showed, people who experienced other stressful events before the COVID-19 pandemic had a higher rate of PTSS and mental health problems.

Consistent with other research results during the COVID-19 pandemic (23, 24), divorced/widowed partici- 


\begin{tabular}{|c|c|c|c|}
\hline Scoring & $\%$ & Mean & SD \\
\hline Total GHQ-28 & & 23.02 & 13.30 \\
\hline$<23$ & 56.4 & & \\
\hline$\geq 23$ & 43.6 & & \\
\hline Somatic symptoms & & 5.83 & 3.95 \\
\hline$\geq 7$ & 36.8 & & \\
\hline Anxiety and sleep disorder & & 5.96 & 4.64 \\
\hline$<7$ & 60.2 & & \\
\hline$\geq 7$ & 39.8 & & \\
\hline Social function & & 7.59 & 3.27 \\
\hline$<7$ & 34.2 & & \\
\hline Depression symptoms & & 3.63 & 4.34 \\
\hline$<7$ & 78.1 & & \\
\hline$\geq 7$ & 21.9 & & \\
\hline Total IES-R & & 29.32 & 17.41 \\
\hline$<24$ & 37.6 & & \\
\hline$\geq 24$ & 62.4 & & \\
\hline Intrusion & - & 10.26 & 7.15 \\
\hline Avoidance & - & 11.65 & 6.68 \\
\hline Hyperarousal & - & 7.40 & 5.77 \\
\hline
\end{tabular}

pants had a significantly higher prevalence of PTSS and mental health burden. This finding may be due to a lack of support against negative emotions and/or lack of opportunities to communicate with family members, which can lead to psychological problems, highlighting the importance of family support in this stressful event (23). Besides, the higher mental health burden in unemployed and lowincome groups in this study, which is in line with other research $(24,25)$, may be related to the negative impacts of economic problems on mental health during the pandemic.

The higher prevalence of the mental health burden and PTSS in women is consistent with other studies $(12,25)$, which may be due to the higher prevalence of known risk factors in women, including chronic environmental stress, preexisting depressive and anxiety disorders, and domestic violence, which is exacerbated during a pandemic (26). In terms of the dimensions of mental health in the present study, the higher prevalence of social dysfunction (65.8\%) than that of anxiety symptoms (39.8\%) and somatic symptoms (36.8\%) may be attributed to the broader effects of the COVID-19 pandemic on individuals and greater impacts on people's social and economic life, especially among adults. About $87.0 \%$ of the population in the current study was in the age range of 18 - 45 years, who probably had worries about the future and were mostly affected by unemployment, inflation, and business closures. On the other hand, the lower prevalence of depression in the present study could be attributed to the short time interval between the onset of the outbreak and the study conduct. Unlike anxiety symptoms, the prevalence of PTSD and depressive symptoms is higher in the late stages and after the epidemic $(27,28)$; thus, follow-up studies are needed to assess the extent of depression and PTSD in Iranians.

People with experience of COVID-19 infection in themselves, their family, or friends may have some concerns about the health condition of the infected person or increased risk of contracting the disease (12); therefore, a higher prevalence of the mental health burden and PTSS in these groups is expected, as shown by this study and other studies $(11,12)$.

The current study revealed a high prevalence of mental health burden and COVID-19-related PTSS in participants who reported a history of psychiatric disorders. People with psychiatric illnesses were more likely to exhibit higher levels of PTSD, depression, anxiety, and stress than people who had no mental illnesses (29). Since outbreaks such as COVID-19 can provoke relapses or even worsen the existing psychological disorder (30), it is essential to support this particular population. Despite the higher prevalence of the mental health burden in healthcare workers in the present study, this population did not have a higher prevalence of PTSS. This may be due to the increased mental preparedness of healthcare workers in the context of their previous experiences, greater access to formal psychological support, more accurate medical information 


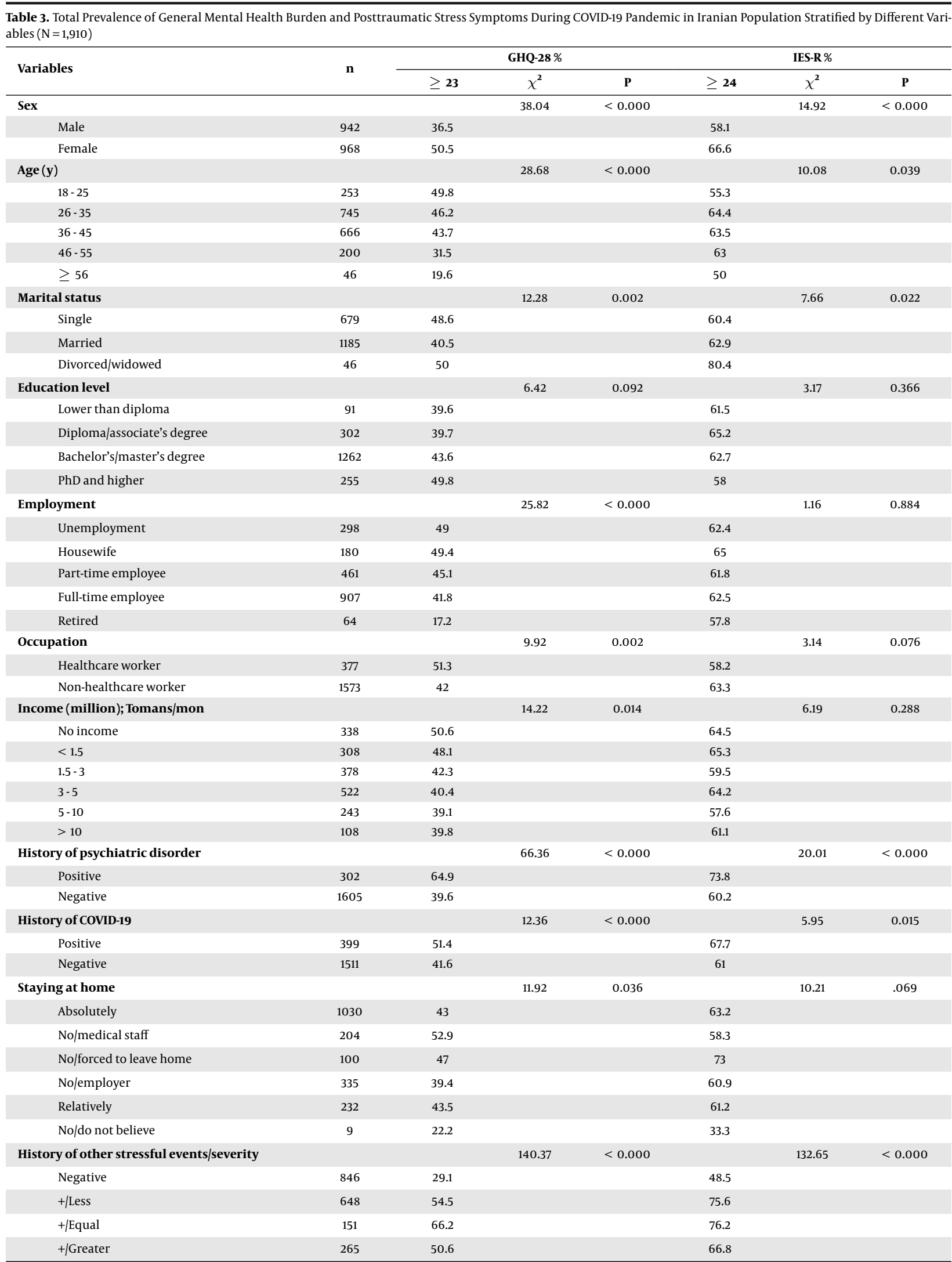


on the pandemic, and better training on personal protective equipment and infection control measures (18).

Multivariable logistic regression analyses showed that the general mental health burden(GHQ-28 $\geq 23$ ) and other stressful events were the potential influencing factors associated with COVID-19-related PTSS. Studies revealed that mental health problems such as anxiety and depression were significant predictive factors of COVID-19-related PTSD (9); also, exposure to other life stressors prior to the index trauma (a trauma that is considered by a person as a stressful event) has been associated with an increased risk of PTSD (22).

Overall, it seems that the COVID-19 pandemic has placed a significant general mental health burden on the Iranian population (43.6\%), especially in the social and anxiety dimensions, leading to PTSS in a significant percentage of participants (62.4\%); on the other hand, the general mental health burden (GHQ-28 $\geq 23$ ) and other stressful events were the potential influencing factors associated with COVID-19-related PTSS. It seems that the rate of mental health involvement during the pandemic is higher in Iran than in other countries considered in such studies. These findings can be used by public health officials to more effectively identify individuals in society who are at higher risk of developing mental health problems in response to the current pandemic. Therefore, in addition to efforts at various levels to prevent the spread of the disease and other worrisome conditions, special attention should be paid to the mental health issues of the community. Moreover, it is important to have some programs for the screening of psychiatric disorders including acute stress disorder or PTSS, anxiety, depression, sleep disorders, and other psychopathologies among the general population, patients, and even caregivers in order to manage and treat the cases by employing psychiatrists, psychologists, and other relevant medical groups.

This study had some limitations. First, the design of this study was cross-sectional, so it is difficult to make any causal inferences. Second, a web-based survey by an online system method was used to avoid the possible spreading of the infection, so the possibility of selection bias should be considered. Also, illiterate people or those who did not have access to social networks could not participate in this study. Therefore, the results may not be generalizable to the whole community. Finally, the relatively small sample size may limit the generalization of the results. Despite these limitations, the current study provides important and new information about the rates of COVID-19-related PTSS and the general mental health burden in the general population.

\subsection{Conclusion}

The study identified a major mental health burden among the public during the COVID-19 pandemic in Iran. Women, people with a history of psychopathology and/or positive history of other stressful events prior to the pandemic were at a high risk of displaying psychological issues. Since the mental health burden is a predictor of PTSS, in order to prevent the long-term consequences such as PTSD, ongoing surveillance and monitoring of the psychological consequences of COVID-19 and early targeted mental health interventions are necessary. Further research is required to delineate the long-term effects of the COVID-19 pandemic on mental health.

\section{Acknowledgments}

The authors would like to thank all participants in our study. In addition, we express our heartfelt respect to all healthcare workers who are fighting the COVID-19 pandemic on the front line.

\section{Footnotes}

Authors' Contribution: Study concept and design, R.S. and M.A.; Acquisition of data, R.S. and M.A.; Analysis and interpretation of data, M.N. and M.A.; Drafting of the manuscript, M.A.; Critical revision of the manuscript for important intellectual content, R.S. and A.G.; Statistical analysis, M.N. and M.A.; Study supervision, R.S.

Conflict of Interests: There are no conflicts of interest.

Ethical Approval: This study was approved by the Ethics Committee of the Iran University of Medical Sciences, Tehran, Iran (code: 1399.011).

Funding/Support: This research did not receive any specific grant from funding agencies in the public, commercial, or not-for-profit sectors.

\section{References}

1. Peeri NC, Shrestha N, Rahman MS, Zaki R, Tan Z, Bibi S, et al. The SARS, MERS and novel coronavirus (COVID-19) epidemics, the newest and biggest global health threats: what lessons have we learned? Int J Epidemiol. 2020;49(3):717-26. doi: 10.1093/ije/dyaa033. [PubMed: 32086938]. [PubMed Central: PMC7197734].

2. Moghadami M, Hassanzadeh M, Wa K, Hedayati A, Malekolkalami M. Modeling the Corona virus outbreak in Iran. medRxiv. 2020;Preprint. doi: 10.1101/2020.03.24.20041095.

3. World Health Organization. Coronavirus disease 2019 (COVID-19): Situation report - 69. Geneva, Switzerland: World Health Organization; 2020.

4. Saladino V, Algeri D, Auriemma V. The psychological and social impact of COVID-19: New perspectives of well-being. Front Psychol. 2020;11:577684. doi: 10.3389/fpsyg.2020.577684. [PubMed: 33132986]. [PubMed Central: PMC7561673]. 
5. Kumar A, Nayar KR. COVID 19 and its mental health consequences. J Ment Health. 2021;30(1):1-2. doi: 10.1080/09638237.2020.1757052. [PubMed: 32339041].

6. Huang Y, Zhao N. Generalized anxiety disorder, depressive symptoms and sleep quality during COVID-19 outbreak in China: A webbased cross-sectional survey. Psychiatry Res. 2020;288:112954. doi: 10.1016/j.psychres.2020.112954. [PubMed: 32325383]. [PubMed Central: PMC7152913].

7. Alkhamees AA, Alrashed SA, Alzunaydi AA, Almohimeed AS, Aljohani MS. The psychological impact of COVID-19 pandemic on the general population of Saudi Arabia. Compr Psychiatry. 2020;102:152192. doi: 10.1016/j.comppsych.2020.152192. [PubMed: 32688022]. [PubMed Central: PMC7354380].

8. Rossi R, Socci V, Talevi D, Mensi S, Niolu C, Pacitti F, et al. COVID19 pandemic and lockdown measures impact on mental health among the general population in Italy. Front Psychiatry. 2020;11:790. doi: 10.3389/fpsyt.2020.00790. [PubMed: 32848952]. [PubMed Central: PMC7426501].

9. Karatzias T, Shevlin M, Murphy J, McBride O, Ben-Ezra M, Bentall RP, et al. Posttraumatic stress symptoms and associated comorbidity during the COVID-19 pandemic in ireland: A population-based study. J Trauma Stress. 2020;33(4):365-70. doi: 10.1002/jts.22565. [PubMed: 32662129]. [PubMed Central: PMC7405473].

10. Salari N, Hosseinian-Far A, Jalali R, Vaisi-Raygani A, Rasoulpoor S, Mohammadi $\mathrm{M}$, et al. Prevalence of stress, anxiety, depression among the general population during the COVID-19 pandemic: A systematic review and meta-analysis. Global Health. 2020;16(1):57. doi: 10.1186/s12992-020-00589-w. [PubMed: 32631403]. [PubMed Central: PMC7338126].

11. Moghanibashi-Mansourieh A. Assessing the anxiety level of Iranian general population during COVID-19 outbreak. Asian J Psychiatr. 2020;51:102076. doi: 10.1016/j.ajp.2020.102076. [PubMed: 32334409]. [PubMed Central: PMC7165107].

12. Yang J, Lv ZY, Jiang HJ, Nan J. Psychological impacts of the COVID-19 epidemic on Chinese people: Exposure, post-traumatic stress symptom, and emotion regulation. Asian Pac JTrop Med.2020;13(6):252. doi: 10.4103/1995-7645.281614.

13. Goldberg DP, Hillier VF. A scaled version of the General Health Questionnaire. Psychol Med. 1979;9(1):139-45. doi: 10.1017/s0033291700021644. [PubMed: 424481].

14. Sterling M. General Health Questionnaire - 28 (GHQ-28). J Physiother. 2011;57(4):259. doi: 10.1016/S1836-9553(11)70060-1. [PubMed: 22093128].

15. Baniasadi M, Divsalar P, Noorbala AA, Eslami Shahrbabaki M, Aflatoonian B, Ashrafi Asgarabad A. Bam earthquake survivors' mental health status 12 years after the earthquake: A population-based study. Arch Iran Med. 2019;22(2):59-64. [PubMed: 30980639].

16. Taghavi S. [Validity and reliability of the General Health Questionnaire (GHQ-28) in college students of Shiraz University]. J Psychol. 2002;5(4):381-98. Persian.

17. Creamer M, Bell R, Failla S. Psychometric properties of the Impact of Event Scale - Revised. Behav Res Ther. 2003;41(12):1489-96. doi: 10.1016/j.brat.2003.07.010. [PubMed:14705607].

18. Tan BYQ, Chew NWS, Lee GKH, Jing M, Goh Y, Yeo LLL, et al. Psychological impact of the COVID-19 pandemic on health care workers in
Singapore. Ann Intern Med. 2020;173(4):317-20. doi: 10.7326/M20-1083. [PubMed: 32251513]. [PubMed Central: PMC7143149].

19. Panaghi L, Hakim Shooshtari. M, Atari Mogadam J. [Persian version validation in impact of event Scale-Revised]. Trans Tehran Univ Med J. 2006;64(52-60). Persian.

20. Noorbala AA, Faghihzadeh S, Kamali K, Bagheri Yazdi SA, Hajebi A Mousavi MT, et al. Mental health survey of the Iranian Adult Population in 2015. Arch Iran Med. 2017;20(3):128-34. [PubMed: 28287805].

21. Suliman S, Mkabile SG, Fincham DS, Ahmed R, Stein DJ, Seedat S. Cumulative effect of multiple trauma on symptoms of posttraumatic stress disorder, anxiety, and depression in adolescents. Compr Psychiatry. 2009;50(2):121-7. doi: 10.1016/j.comppsych.2008.06.006. [PubMed: 19216888].

22. Sareen J. Posttraumatic stress disorder in adults: Impact, comorbidity, risk factors, and treatment. Can J Psychiatry. 2014;59(9):460-7. doi: 10.1177/070674371405900902. [PubMed: 25565692]. [PubMed Central: PMC4168808].

23. Liang L, Ren $\mathrm{H}$, Cao R, Hu Y, Qin Z, Li C, et al. The effect of COVID-19 on youth mental health. Psychiatr Q. 2020;91(3):841-52. doi: 10.1007/s11126-020-09744-3. [PubMed: 32319041]. [PubMed Central: PMC7173777].

24. Holingue C, Badillo-Goicoechea E, Riehm KE, Veldhuis CB, Thrul J, Johnson RM, et al. Mental distress during the COVID-19 pandemic among US adults without a pre-existing mental health condition: Findings from American trend panel survey. Prev Med. 2020;139:106231. doi: 10.1016/j.ypmed.2020.106231. [PubMed: 32758507]. [PubMed Central: PMC7846292].

25. Pieh C, Budimir S, Probst T. The effect of age, gender, income, work, and physical activity on mental health during coronavirus disease (COVID-19) lockdown in Austria.J Psychosom Res. 2020;136:110186. doi: 10.1016/j.jpsychores.2020.110186. [PubMed: 32682159]. [PubMed Central: PMC7832650].

26. Almeida M, Shrestha AD, Stojanac D, Miller LJ. The impact of the COVID-19 pandemic on women's mental health. Arch Womens Ment Health. 2020;23(6):741-8. doi: 10.1007/s00737-020-01092-2. [PubMed: 33263142]. [PubMed Central: PMC7707813].

27. Liu X, Kakade M, Fuller CJ, Fan B, Fang Y, Kong J, et al. Depression after exposure to stressful events: Lessons learned from the severe acute respiratory syndrome epidemic. Compr Psychiatry. 2012;53(1):15-23. doi: 10.1016/j.comppsych.2011.02.003. [PubMed: 21489421]. [PubMed Central: PMC3176950].

28. Mak IW, Chu CM, Pan PC, Yiu MG, Chan VL. Long-term psychiatric morbidities among SARS survivors. Gen Hosp Psychiatry. 2009;31(4):31826. doi: 10.1016/j.genhosppsych.2009.03.001. [PubMed: 19555791]. [PubMed Central: PMC7112501].

29. Hao F, Tan W, Jiang L, Zhang L, Zhao X, Zou Y, et al. Do psychiatric patients experience more psychiatric symptoms during COVID-19 pandemic and lockdown? A case-control study with service and research implications for immunopsychiatry. Brain Behav Immun. 2020;87:1006. doi: 10.1016/j.bbi.2020.04.069. [PubMed: 32353518]. [PubMed Central: PMC7184991].

30. Ellepola A, Rajapakse RP. Foreseeable psychological impact of COVID-19 in Sri Lanka. ResearchGate. 2020;Preprint. doi: 10.13140/RG.2.2.26156.21120 
Table 4. Binary Logistic Regression Results Predicting General Mental Health Burden and Posttraumatic Stress Symptoms ${ }^{\text {a }}$

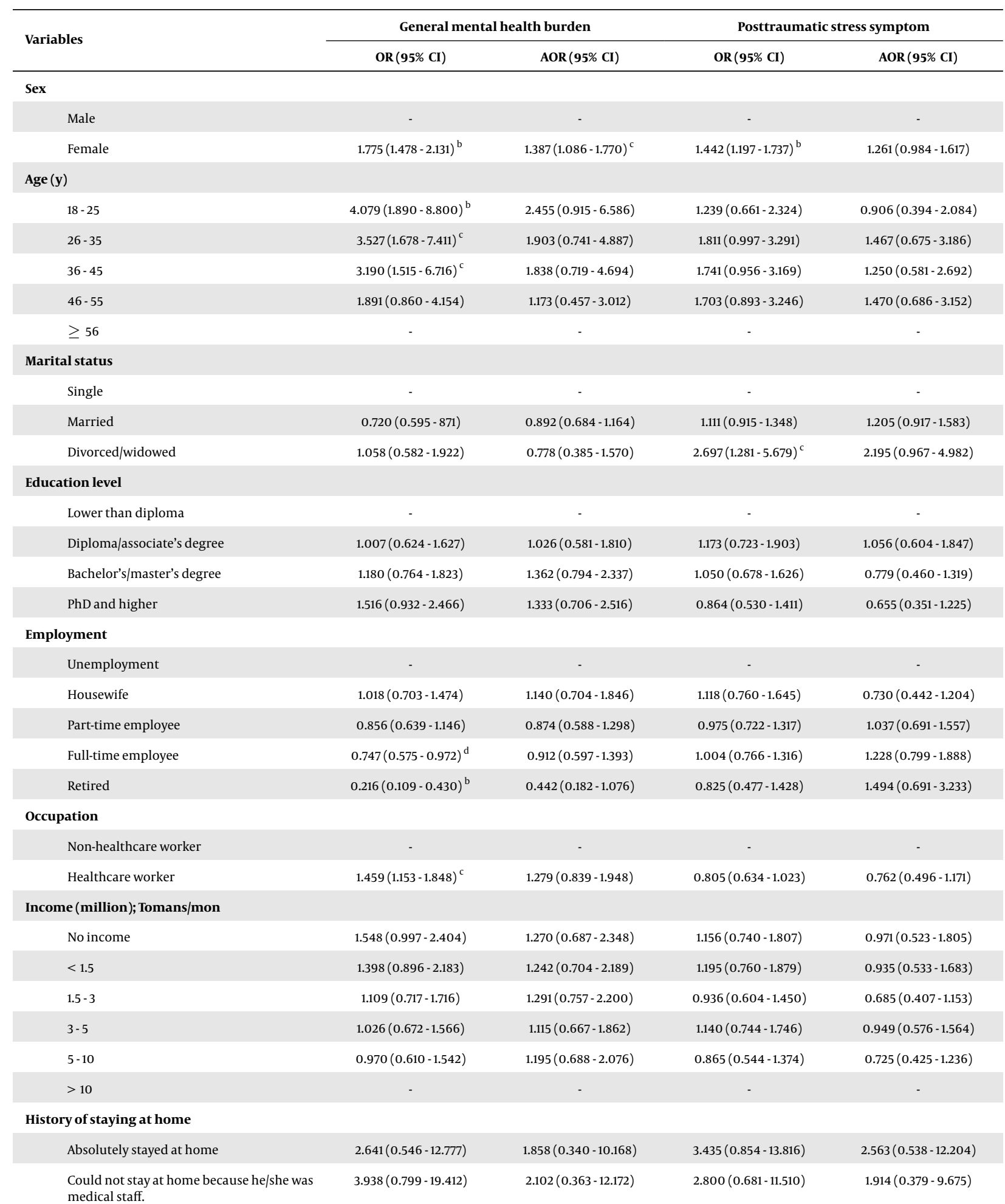




\begin{tabular}{|c|c|c|c|c|}
\hline $\begin{array}{l}\text { Forced to leave home due to financial } \\
\text { problems }\end{array}$ & $3.104(0.614-15.680)$ & $1.995(0.345-11.520)$ & $5.407(1.263-23.157)^{d}$ & $3.667(0.718-18.727)$ \\
\hline $\begin{array}{l}\text { Could not stay at home because he/she was } \\
\text { an employer }\end{array}$ & $2.276(0.466-11.123)$ & $1.919(0.344-10.700)$ & $3.115(0.766-12.670)$ & $2.263(0.466-10.999)$ \\
\hline Relatively stayed at home & $2.698(0.549-13.269)$ & $1.915(0.344-10.645)$ & $3.156(0.770-12.936)$ & $2.348(0.485-11.377)$ \\
\hline She/he did not believe in staying at home & - & - & - & - \\
\hline \multicolumn{5}{|l|}{ History of psychiatric disorder } \\
\hline Negative & - & - & - & - \\
\hline Positive & $2.825(2.185-3.651)^{\mathrm{b}}$ & $2.266(1.680-3.058)^{b}$ & $1.862(1.414-2.453)^{\mathrm{b}}$ & $1.156(0.838-1.593)$ \\
\hline \multicolumn{5}{|l|}{ History of COVID-19 } \\
\hline Negative & - & - & - & - \\
\hline Positive & $1.486(1.191-1.854)^{\mathrm{b}}$ & $1.215(0.936-1.577)$ & $1.337(1.058-1.689)^{d}$ & $1.178(0.899-1.545)$ \\
\hline \multicolumn{5}{|l|}{ History of other stressful events/severity } \\
\hline Negative & - & - & - & - \\
\hline$+/$ Less $^{e}$ & $2.919(2.356-3.616)^{\mathrm{b}}$ & $1.978(1.555-2.517)^{\mathrm{b}}$ & $3.298(2.635-4.127)^{\mathrm{b}}$ & $2.482(1.938-3.179)^{\mathrm{b}}$ \\
\hline+ Equal $^{\mathrm{f}}$ & $4.782(3.308-6.913)^{\mathrm{b}}$ & $2.966(1.977-4.449)^{\mathrm{b}}$ & $3.397(2.282-5.057)^{\mathrm{b}}$ & $2.080(1.339-3.230)^{\mathrm{C}}$ \\
\hline$+/$ Greater $^{g}$ & $2.495(1.880-3.311)^{\mathrm{b}}$ & $1.713(1.238-2.370)^{\mathrm{C}}$ & $2.139(1.602-2.856)^{b}$ & $1.803(1.296-2.508)^{b}$ \\
\hline \multicolumn{5}{|l|}{ PTSS } \\
\hline IES-R $<24$ & - & - & & \\
\hline IES-R $\geq 24$ & $6.587(5.271-8.231)^{\mathrm{b}}$ & $6.013(4.725-7.653)^{\mathrm{b}}$ & & \\
\hline \multicolumn{5}{|l|}{ General mental health burden } \\
\hline GHQ- $28<23$ & & & - & - \\
\hline GHQ-28 $\geq 23$ & & & $6.587(5.271-8.231)^{\mathrm{b}}$ & $6.007(4.722-7.642)^{b}$ \\
\hline
\end{tabular}

Abbreviations: OR, odds ratio; AOR, adjusted odds ratio (multivariable with IES-R and GHQ-28 score included).

${ }^{\mathrm{a}} \mathrm{N}=1,910$.

${ }^{\mathrm{b}} \mathrm{P}<0.001$.

${ }^{\mathrm{c}} \mathrm{P}<0.01$.

${ }^{\mathrm{d}} \mathrm{P}<0.05$.

e Positive but its distress severity was less than that of COVID-19 pandemic.

${ }^{\mathrm{f}}$ Positive and its distress severity was equal to that of COVID-19 pandemic.

${ }^{g}$ Positive but its distress severity was greater than that of COVID-19 pandemic. 